\title{
Paroi tirantée et clouée à Beyrouth
}

J.-B. ESTA

Ingénieur Civil des Ponts et Chaussées, Professeur à titre personnel

à l'École supérieure d'ingénieurs de Beyrouth
L'auteur expose le confortement par clouage d'une des fouilles les plus profondes exécutées dans Beyrouth de l'après-guerre.

Au clouage proprement dit ont été associés des tirants précontraints pour limiter les déplacements en tête de la paroi au droit de laquelle existent des bâtiments de 9 à 15 étages.

\section{Anchored and nailed facing at Beirut}

The author exposes the retaining by nailing to one of the leepest excavation executed in Beirut of the after war (max. height $23 \mathrm{~m}$ )

To the properly so called nailing, prestressed anchors were associated to limit any deformations at the top of the nailed facing where 9 to 15 storied buildings exist. 


\section{Introduction}

La destruction du centre de Beyrouth durant la guerre de 1975-1990 a provoqué l'émergence de plusieurs agglomérations commerciales de part et d'autre de la ligne de démarcation qui coupait la ville. Au lendemain de l'arrêt des combats en 1990 un nouveau plan fut établi pour la reconstruction du centre de Beyrouth dont l'achèvement était programmé au mieux pour l'an 2010. Cela favorisa encore plus le développement des agglomérations commerciales créées durant la guerre, et un effort certain fut développé dans ces zones : la rue Verdun à l'ouest de Beyrouth en est un exemple frappant.

Par ailleurs les lois de l'urbanisme aidant, les nouveaux immeubles commerciaux furent dotés de soussols profonds, et rares sont ceux qui, construits après 1990, n'en possèdent au moins quatre.

Toutefois, leur mitoyenneté avec des immeubles plus ou moins récents comportant au plus trois soussols entraine des frais supplémentaires souvent importants pour le soutènement qu'il convient de minimiser.

L.e cas exposé ici est celui du Centre Verdun 732 situé sur la rue Verdun. Ce Centre doit être construit sur une parcelle de $80 \times 40 \mathrm{~m}^{2}$ et comprend à l'arrière une tour de 18 étages et, sur la rue elle-même, quatre étages commerciaux, les sous-sols étant au nombre de six.

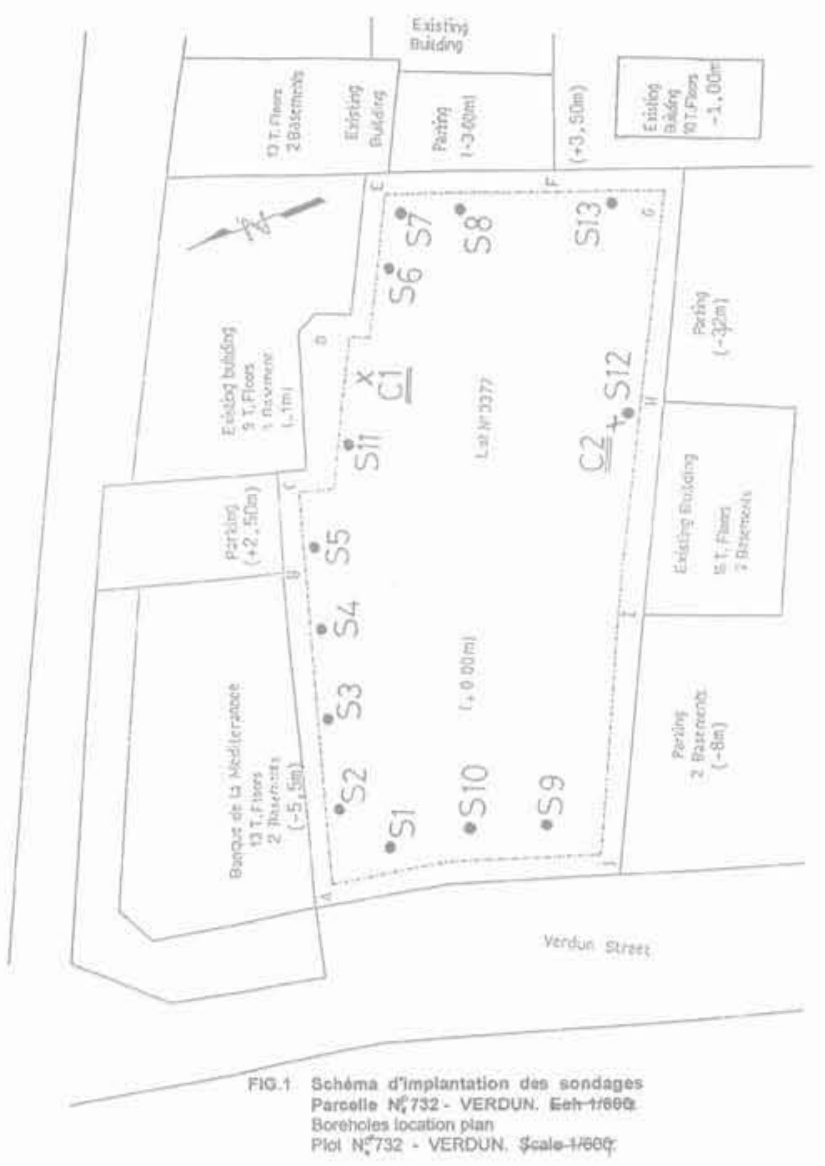

FIG 1 Schéma d'implantation des sondages Parcelle N. 732 - VERDUN. Ech 1/600. Boreholes location plan Plot N. 732 - VERDUN. Scale $1 / 600$
La profondeur de l'excavation à réaliser est donc de $18 \mathrm{~m}$ par rapport à la rue principale, mais elle peut atteindre $22 \mathrm{~m}$ sur certains troncons ; par ailleurs, la fouille est bordée sur trois de ses faces par des bâtiments de 9 à 16 étages avec 1 à 3 sous-sols ( -1 à 7 ) ou des parkings de surface $(+3 \mathrm{~m})$ ou en sous-sol (juscu'à $-10 \mathrm{~m}$ ). La figure 1 donne le plan et situe les différents bâtiments existant sur le pourtour de la parcelle.

2

\section{Contexte géologique}

D'après Dubertret le relief principal de la plateforme de Beyrouth est la croupe, en grande partie calcaire et marneuse, qui porte la ville. Cette croupe se compose des deux buttes d'Achrafié et de Ras Beyrouth qui se font face le long d'une cassure se développant sur l'actuelle rue des Banques.

Dans la butte de Ras Beyrouth, on distingue un socle cénomanien calcaire à large sommet de $80 \mathrm{~m}$ d'altitude et un manteau de dunes qui couvrent la partie Est de la butte où se trouve la rue Verdun. Ces dunes se sont en partie figées et transformées en ramleh et s'élèvent, sous cette forme, jusqu'à $100 \mathrm{~m}$ d'altitude. Elles dominent donc de $20 \mathrm{~m}$ le socle cénomanien.

Toutefois, ces dunes ont pu subir, parfois sur de grandes étendues, une décalcification si avancée qu'il n'en subsiste que des sols rouges surtout en surface. En profondeur les vieilles dunes fixées par cimentation de leurs grains par du calcaire se trouvent encore sous une forme plus ou moins compacte. Dans le cas où la cimentation est très poussée, le grès est appelé ramleh. Notons que le ramleh de bonne qualité a servi, au début de ce siècle, à la construction de la majeure partie des immeubles à Beyrouth.

\section{3}

\section{Données sur la coupe du sol}

Les données géotechniques ont été déterminées en deux campagnes réalisées la première par ASSACO à partir de cinq sondages carottés de $30 \mathrm{~m}$ à $40 \mathrm{~m}$ de profondeur chacun et la seconde par SOIL MECHANICS à partir de deux sondages carottés de $20 \mathrm{~m}$ de profondeur avec essais pressiométriques et treize pénétrations statiques au moyen du pénétromètre ANDINA qui ont atteint le refus entre 5 et $10 \mathrm{~m}$ de profondeur.

La stratigraphie du site est assez homogène et comporte:

- en surface 2 à $4 \mathrm{~m}$ de sable silteux lâche ou des remblais avec une résistance de pointe inférieure à $5 \mathrm{MPa}$; - en profondeur, jusqu'à $20 \mathrm{~m}$ de profondeur, des sables plus ou moins cimentés très denses avec des lentilles gréseuses sur lesquelles le refus du pénétromètre est atteint.

Toutefois, la tendance est à l'amélioration en fonction de la profondeur car aucune pénétration n'a pu dépasser les $10 \mathrm{~m}$, d'une part, et d'autre part, à partir de 12-13 m les valeurs des pressions limites et des modules sont élevées, de l'ordre de $3 \mathrm{MPa}$ et plus pour les premières et de 30 à $50 \mathrm{MPa}$ pour les secondes; - au-delà, le substratum est constitué de marne crayeuse très compacte. 

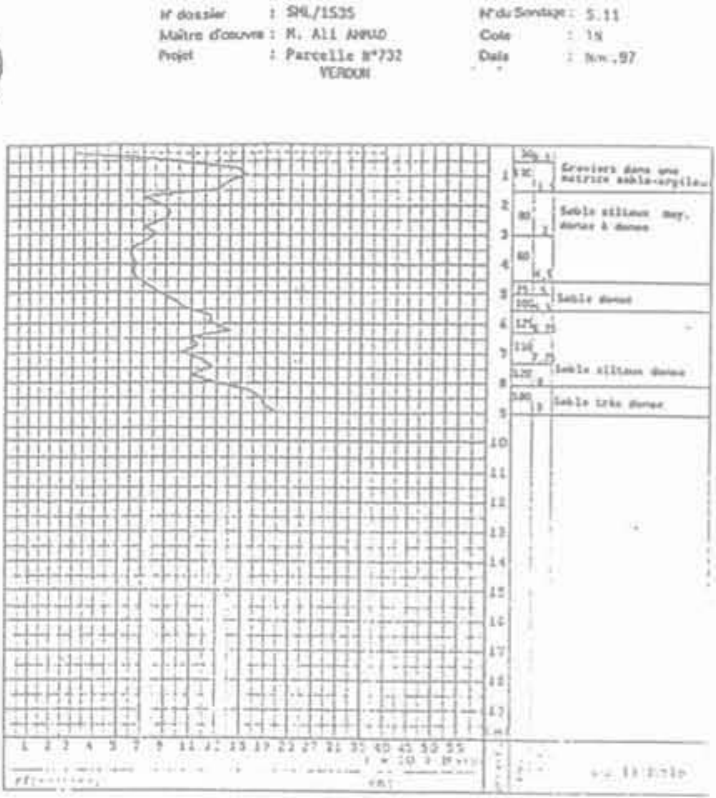

FEUTLLE D $\cong$ SONDAGE

(a)
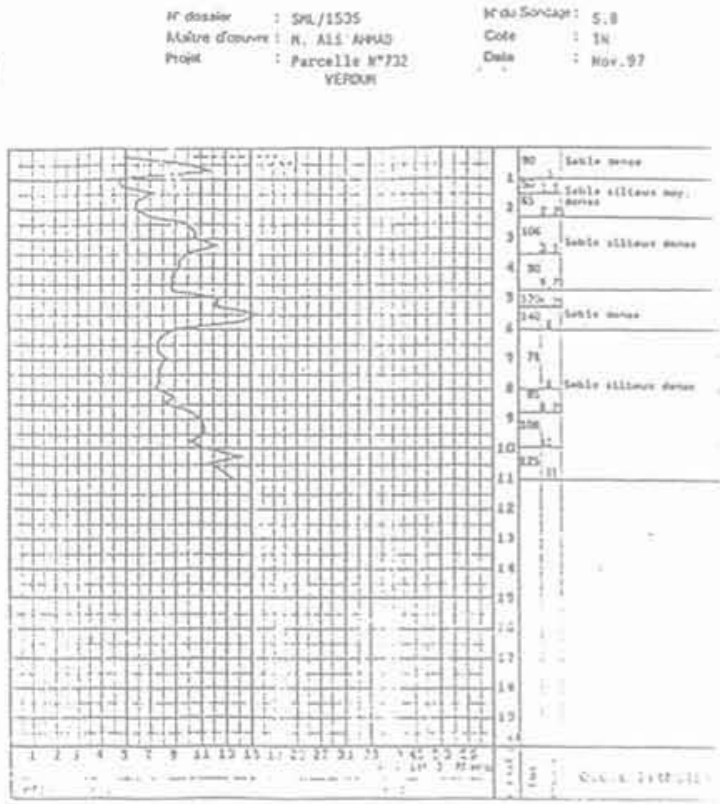

FIG. 2 Sondages S.3, S.8 et S.11. Boreholes S.3, S.8 and S.11.

\section{4}

\section{Données sur les paramètres du sol}

Les échantillons de sable ont été soumis à des essais de cisaillement à la boite qui ont donné un angle de frottement interne compris entre $34^{\circ}$ et $36^{\circ}$ avec des cohésions comprises entre 0 et $6 \mathrm{kPa}$. Cela est normal pour des sables légèrement cimentés qui ne permettent pas un prélèvement d'échantillon sans destruction plus ou moins partielle de cette cimentation.

Toutefois, l'analyse fine des courbes pénétrométriques statiques indique qu'il existe une cimentation
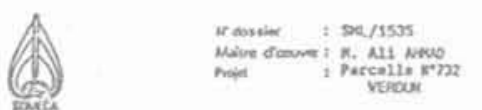

rousondwe: 5.3

(a) :

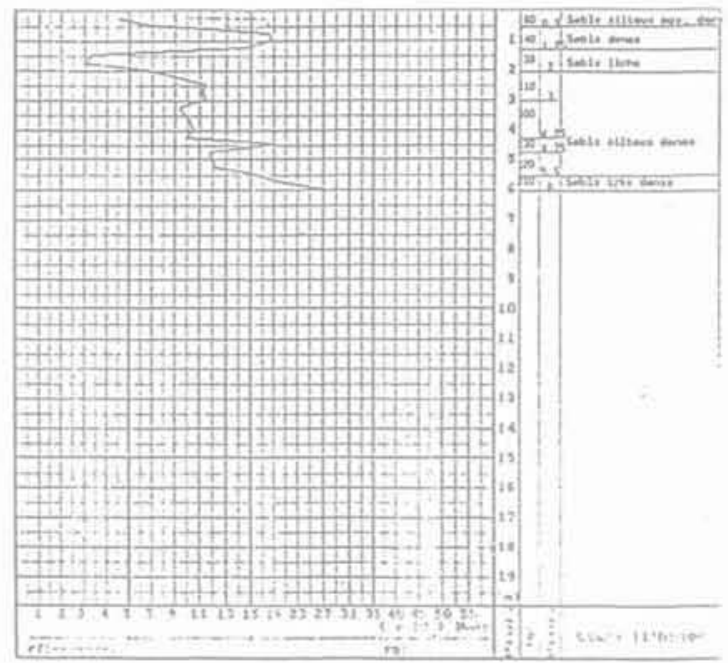

donnant une cohésion supérieure à $10 \mathrm{kPa}$ car pour des résistances de pointe relativement élevées, on observe des segments verticaux sur le tracé des valeurs de $q_{c}$ qui indiquent le passage dans des strates cohérentes (Fig. 2).

Ces interprétations conduisent à retenir, pour le calcul du soutenement, les caractéristiques suivantes :

- de 0 à 2 ou $4 \mathrm{~m}$ suivant le cas :

$\begin{array}{lll}\text { - de } 2 \text { ou } 4 \text { m à } 9 \mathrm{~m} & \varphi=32^{\circ} & \mathrm{c}=10 \mathrm{kPa} ; \\ \text { - au-delà de } 9 \mathrm{~m} & \varphi=32^{\circ} & \mathrm{c}=30 \mathrm{kPa} ; \\ & \varphi=32^{\circ} & \mathrm{c}=45 \mathrm{kPa} .\end{array}$

En cours d'excavation on découvrit que la cohésion de $30 \mathrm{kPa}$ était justifiée et sans doute du côté de la sécurité par l'observation et l'analyse du phénomène suivant:

Le chantier ayant été divisê en deux pour les impératifs de circulation des engins de transport des matériaux excavés, on fut amené dans la zone non concernée par l'excavation à tailler le massif sableux sur une dizaine de mètres de hauteur pratiquement à la verticale, et ce talus est resté stable pendant au moins deux mois avant que l'on vienne le terrasser pour poursuivre le chantier.

Une analyse de stabilité globale montre que cela ne peut se produire que si la cohésion est au moins égale à $60 \mathrm{kPa}$, c'est-à-dire le double de celle adoptée,

D'où vient cette cohésion? La figure 3 montre que le massif est constitué de sable peu cohérent armé par des strates cimentées de 3 à $5 \mathrm{~cm}$ d'épaisseur à la manière d'une terre armée, ce qui confère au massif sableux qui s'écroulerait sans leur présence une cohésion globale lui permettant des performances plus importantes.

Par ailleurs on a adopté, sur la base des abaques de Bustamante, les frottements latéraux sol/inclusion suivants :

- sables silteux de surface:

- sables cimentés jusqu'à $9 \mathrm{~m}$ :

qs $=100 \mathrm{kPa}$;

- sables cimentés au-delà de $9 \mathrm{~m}$. 


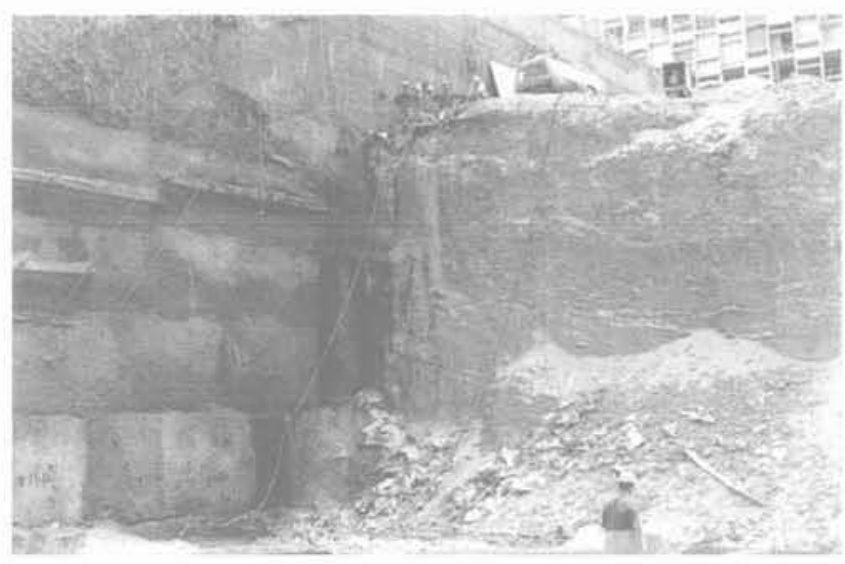

FiG.3 Vue du massif sableux armé par des strates gréseuses.

View of the sandy massif armed by the sandstone strata.

La première valeur est celle que l'on trouve dans CLOUTERRE 91, alors que les deux autres sont légèrement supérieures à celles adoptées dans ce document, mais ont été justifiées par les données que nous possédions sur ce type de sol en exécutant le confortement de la fouille de Verdun 730 juste de l'autre côté de la rue et a posteriori par les observations ci-dessus décrites.

\section{Étude géotechnique}

La présence d'immeubles de grande hauteur ceinturant la parcelle et la profondeur importante de la fouille rendaient le soutènement indispensable.

La première solution classique étudiée était constituée de pieux $\Phi 80$, d'entr'axe plus ou moins grand, retenus par trois nappes de tirants précontraints. La proposition faite par SOLS RENFORCÉS de réaliser un clouage avec un parement en béton projeté de $20 \mathrm{~cm}$ d'épaisseur était alléchante pour le maitre d'œuvre, car I'économie réalisée par la réduction de l'espace perdu de $80 \mathrm{~cm}$ à $20 \mathrm{~cm}$ était de l'ordre de grandeur du coût des travaux proposés.

Toutefois, l'environnement sensible du projet et l'absence de références au Liban de fouilles aussi exceptionnelles conduisirent le maître d'œuvre à exiger l'examen de la fiabilité de la solution par Terrasol et son contrôle par APAVE.

Ce bureau d'étude et ce bureau de contrôle validèrent les choix des paramètres géomécaniques adoptés par SOIL MECHANICS et conclurent que la conception et le dimensionnement des ouvrages proposés par SOLS RENFORCÉS étaient conformes aux Règles de l'Art.

Toutefois, vu le caractère exceptionnel de l'ouvrage par sa hauteur et par son environnement sensible, TERRASOL exigea :

- « des essais préalables d'arrachement de clous;

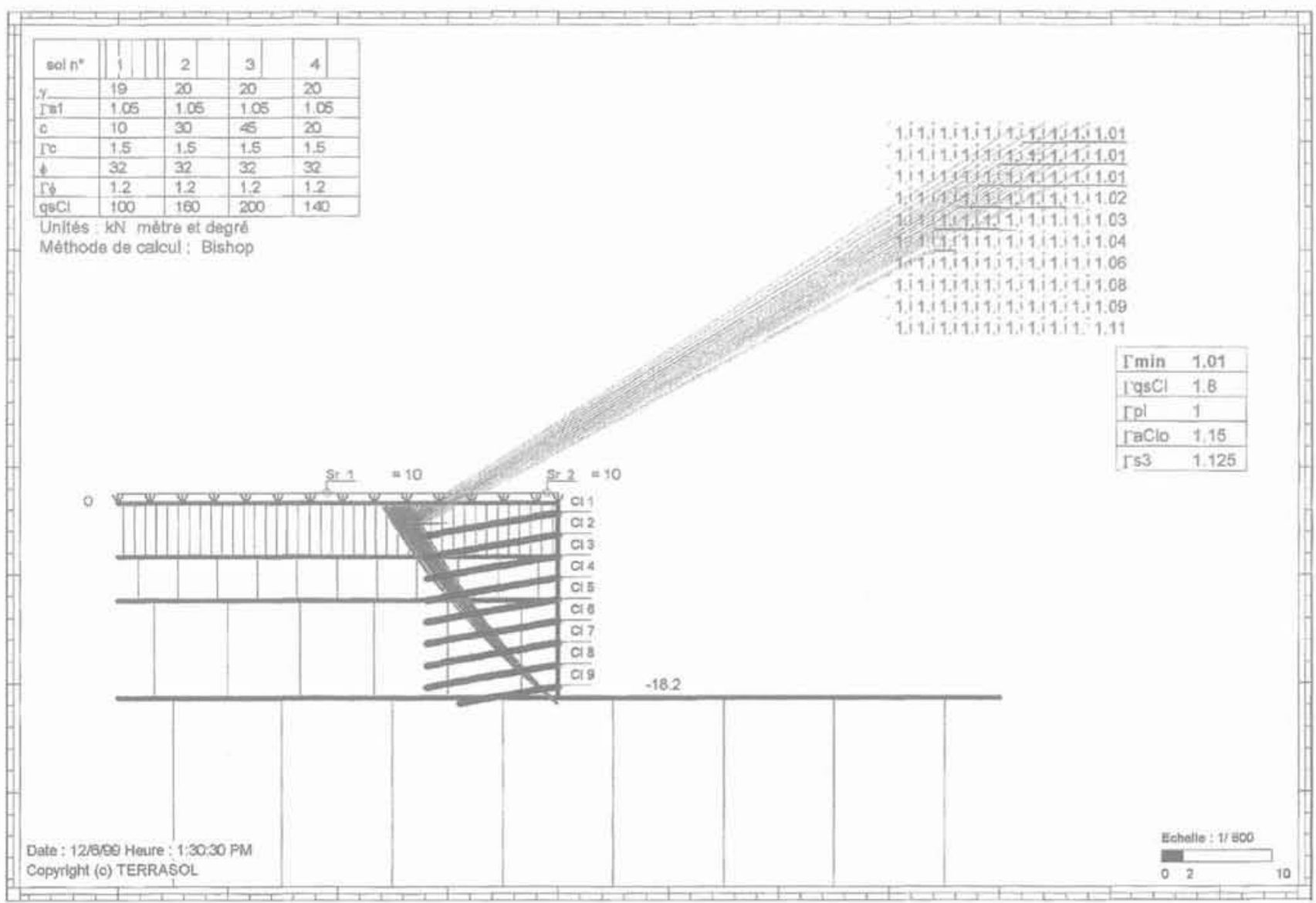



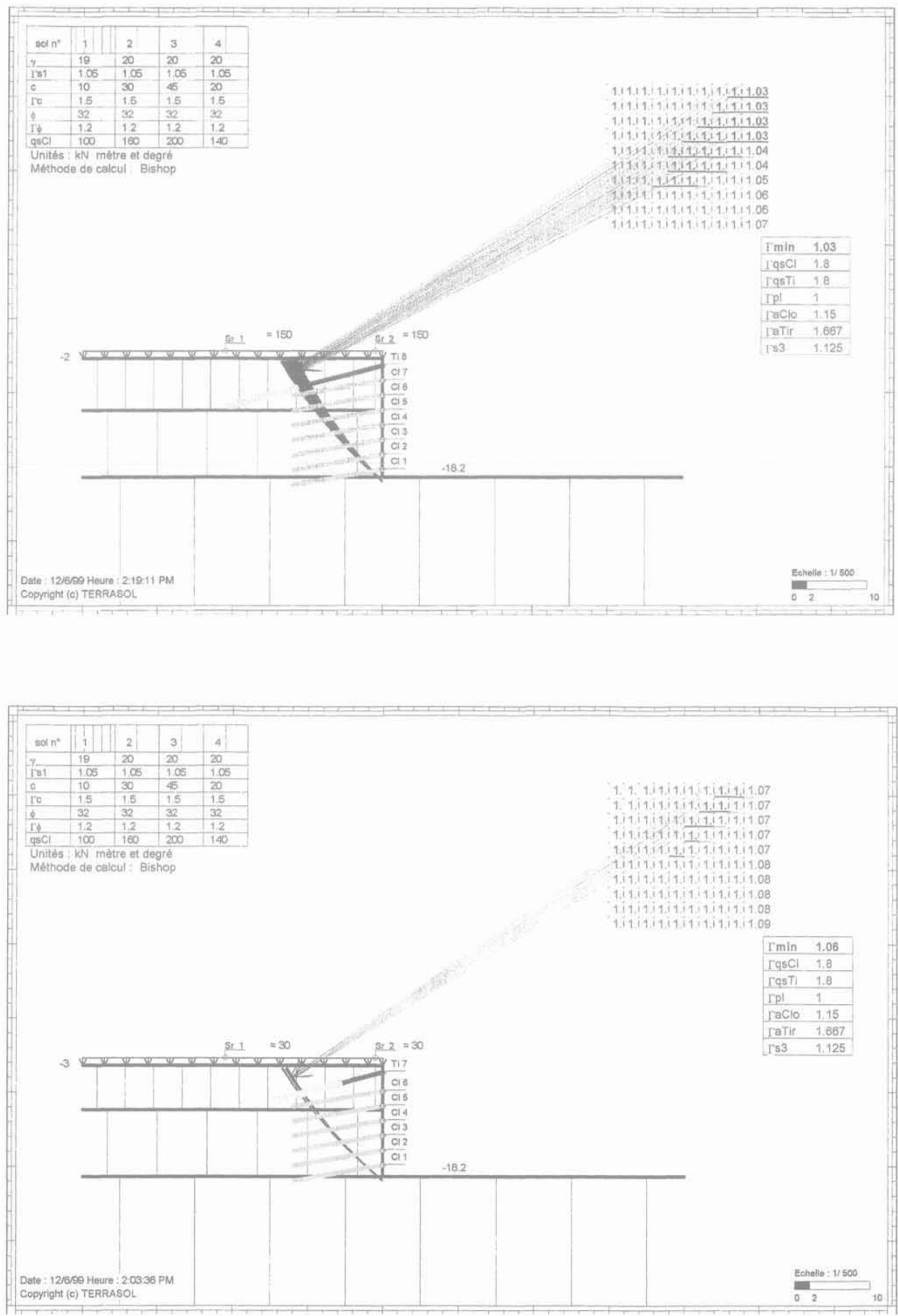

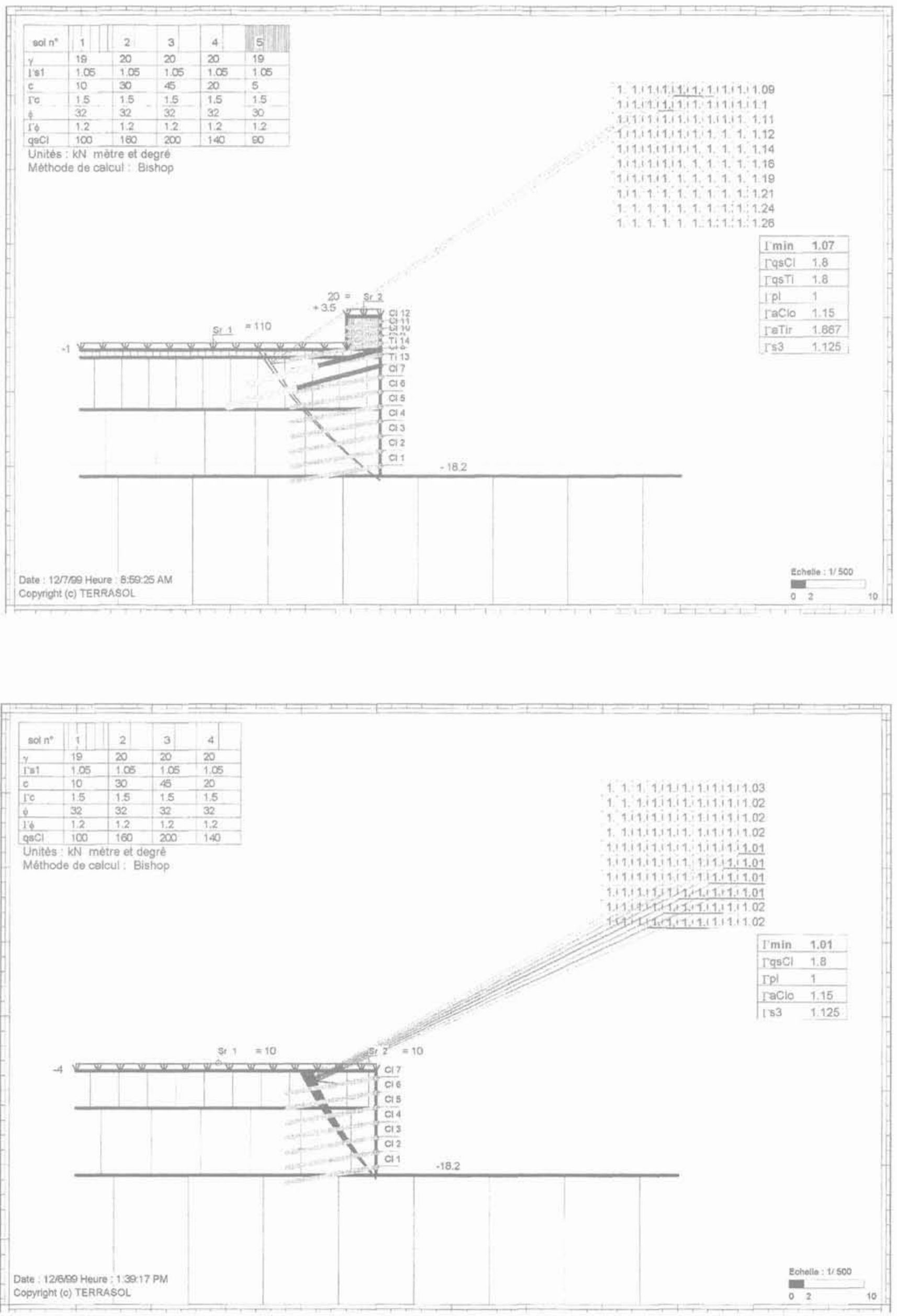

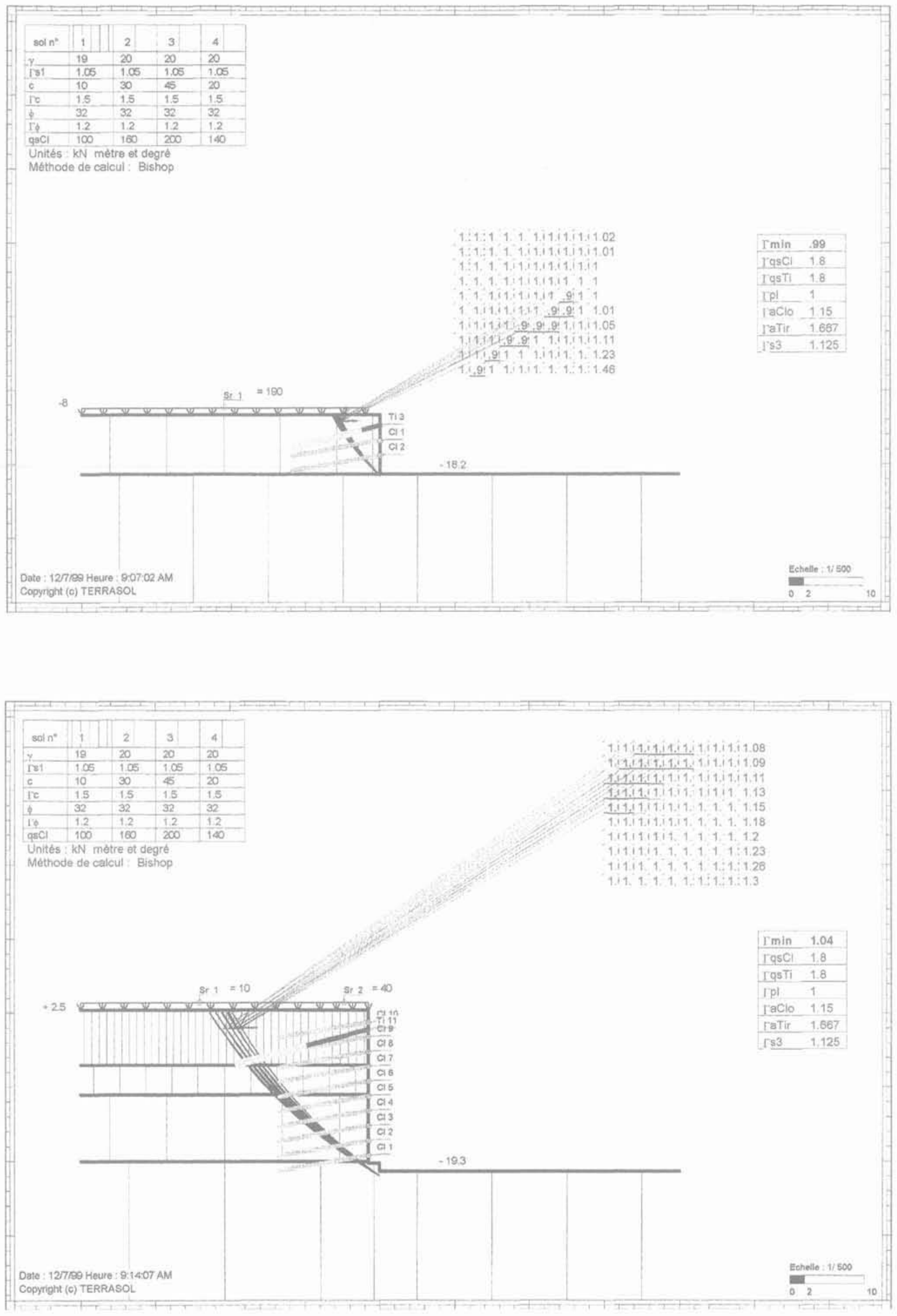


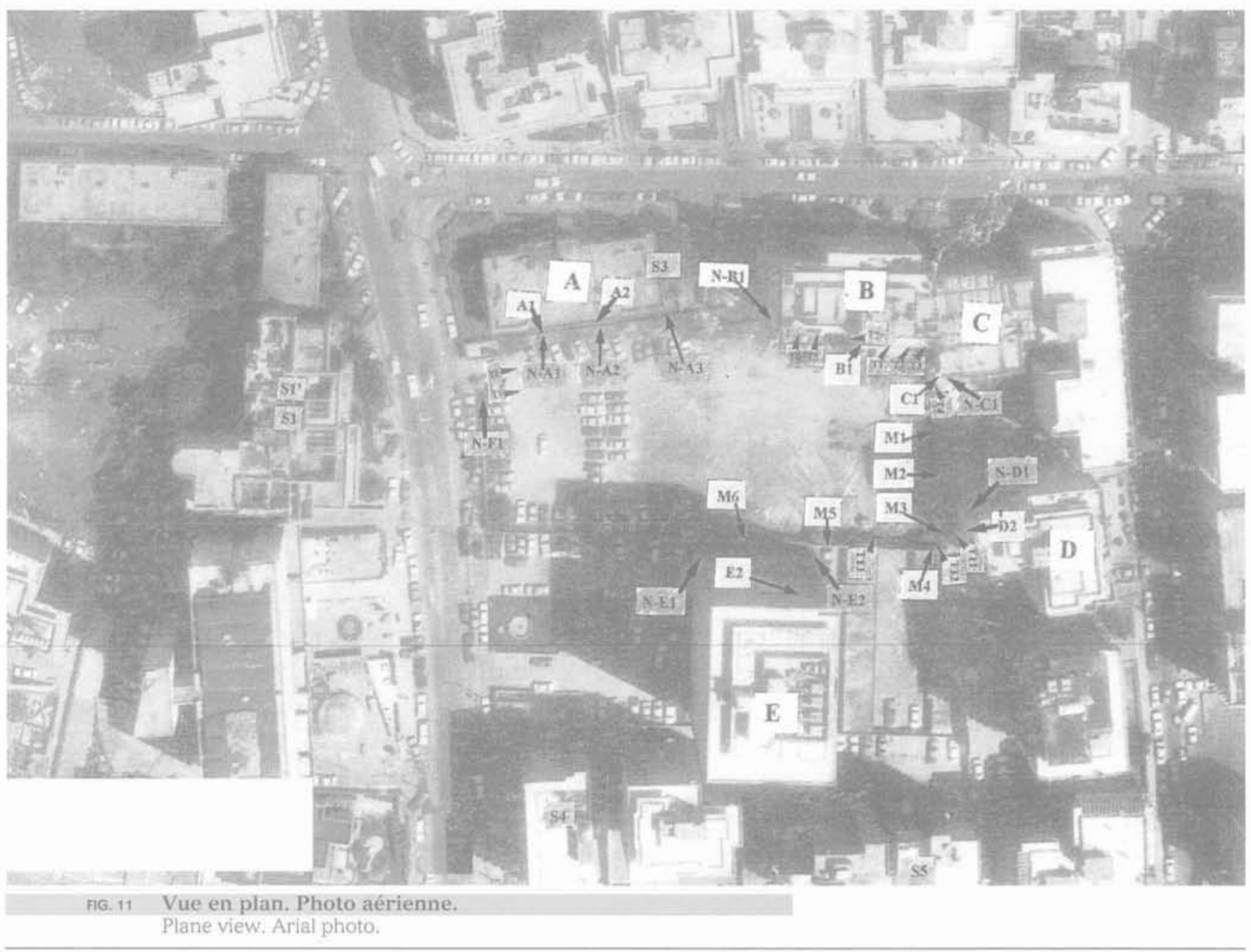

\begin{tabular}{|c|c|c|c|c|c|}
\hline sol $n^{*}$ & 1 & 2 & 3 & 4 & 5 \\
\hline$\gamma$ & 19 & 20 & 20 & 20 & 19 \\
\hline$\sqrt{81}$ & 1.05 & 1.05 & 1.05 & 1.06 & 1.05 \\
\hline c & 10 & 30 & 45 & 20 & S \\
\hline$\Gamma 0$ & 1.6 & 1.5 & 1.5 & 1.5 & 1.5 \\
\hline 6 & 32 & 32 & 32 & 32 & 30 \\
\hline 10 & 1.2 & 12 & 1.2 & 1.2 & 1.2 \\
\hline $98 \mathrm{Cl}$ & 100 & 100 & 200 & 140 & 80 \\
\hline
\end{tabular}

gc gc 3igc 3 . 7 iz 9 oc 998

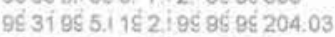

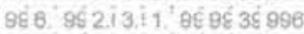

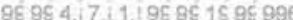

11 oc oć $2:$ oć oc oc oc oc oge

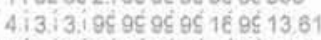

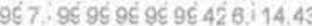

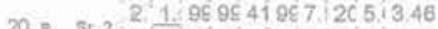

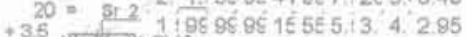

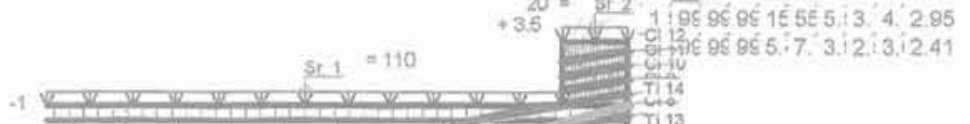

\begin{tabular}{|ll|}
\hline rmin & 1.54 \\
\hline rgsCl & 1.8 \\
\hline rasTi & 1.8 \\
\hline rpl & 1 \\
\hline raClo & 1.15 \\
\hline raTir & 1.687 \\
\hline rs3 & 1.125 \\
\hline
\end{tabular}

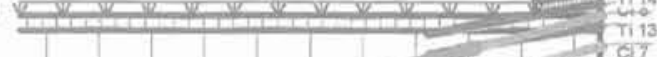


- un contrôle très strict des passes de terrassement; - une auscultation soignée des immeubles voísins avec des mesures topographiques des mouvements horizontaux et verticaux $)$.

La solution approuvée était ainsi conçue :

- Immédiatement sous le niveau des fondations, au plus de $1 \mathrm{~m}$ en dessous, une nappe de tirants précontraints longs $18 \mathrm{~m}$ à $21 \mathrm{~m}$ suivant le cas, pour bloquer le plus possible les déplacements en tête inhérents à la technique du clouage, de l'ordre de $\mathrm{H} / 1000$ pour les sols raides d'après CLOUTERRE 91, H étant la hauteur de la fouille. Cela aurait conduit, sans les tirants, à des tassements et à des déplacements horizontaux de la crête du mur de l'ordre de $18 \mathrm{~mm}$ et qui s'atténueraient sur une distance de $0,8 \mathrm{H}$ soit environ $15 \mathrm{~m}$.

Bien que les déformations différentielles qui en auraient résultê, de l'ordre de 1,3/1 000, étaient acceptables pour les bâtiments en béton armé courants, on a préféré limiter l'effet de ces déformations par la mise en place de cette nappe de tirants précontraints.

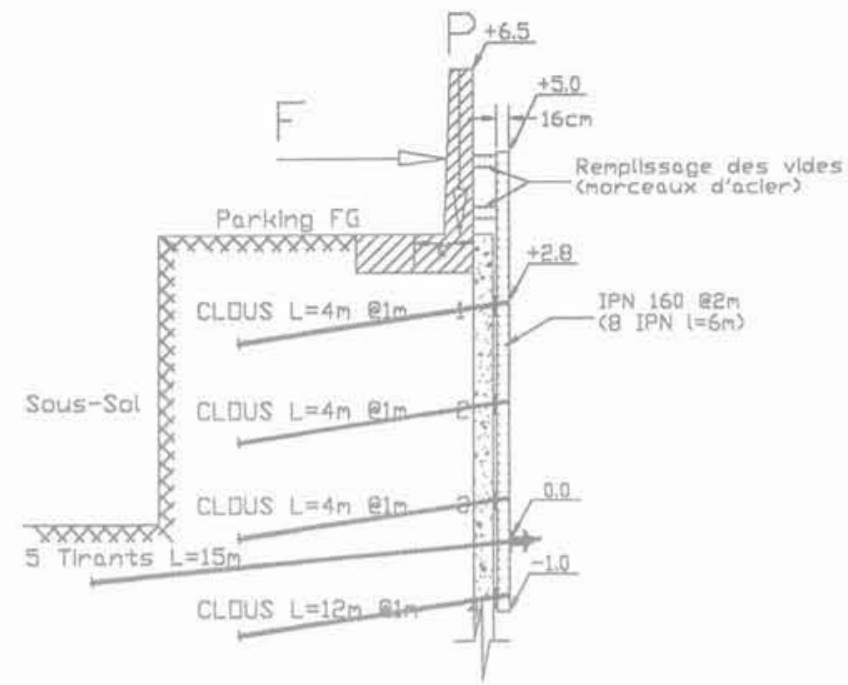

FG.13 Renforcement du côté FG. Coupe - Éch $1 / 100$.

Reinforcement of side FG. Section - Scale 1/100.

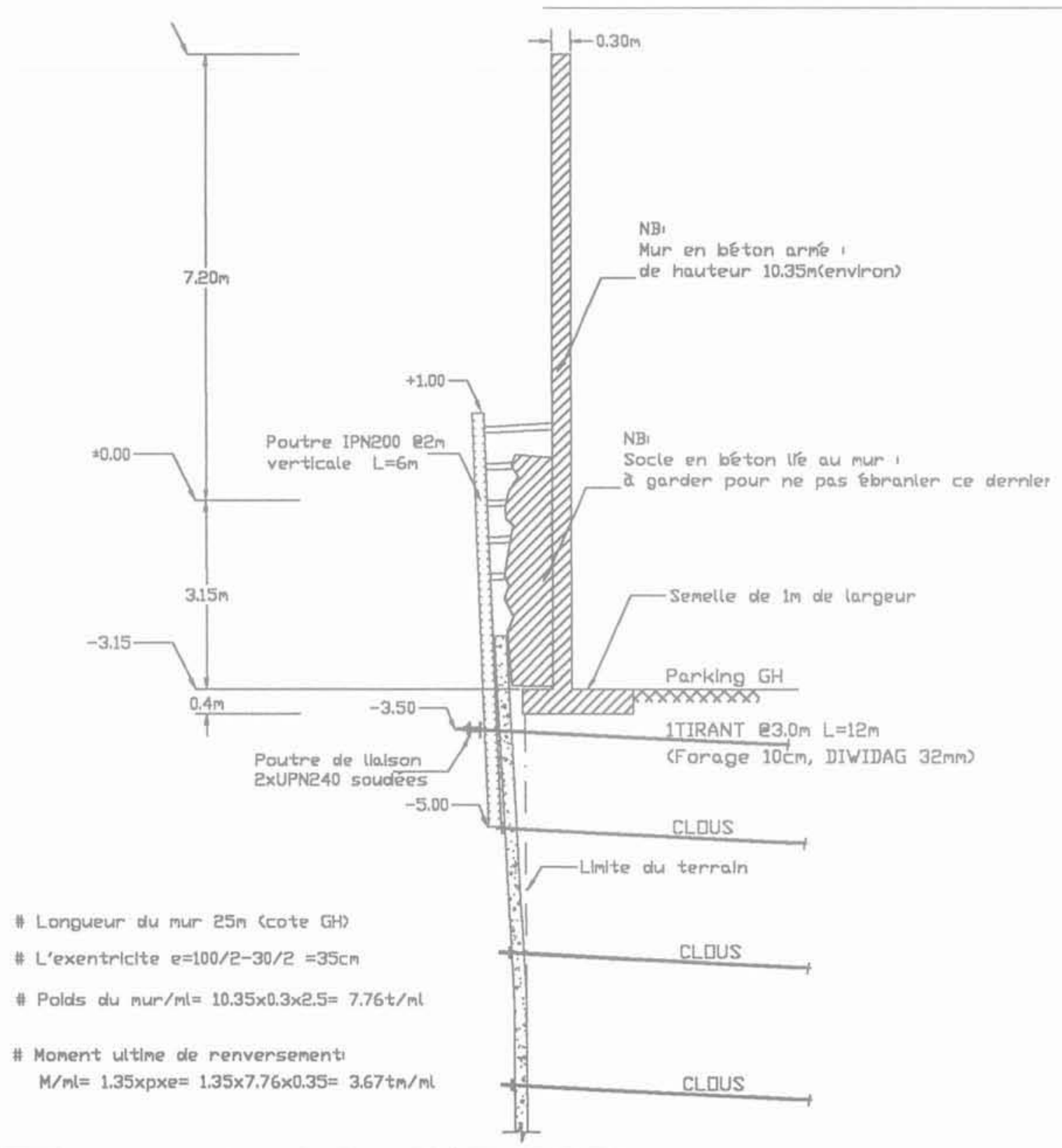

FIG. 14 Renforcement du mur de clôture. Côté GH - VERDUN 732.

Reinforcement of fence wall. Side GH - VERDUN 732 
Il convient de noter que les tirants précontraints que nous utilisons depuis vingt ans sont des monobarres DYWIDAG de $32 \mathrm{~mm}$ de diamètre, de nuance 900/1030, dont la charge à la limite élastique est égale à $724 \mathrm{kN}$. En tête de mur, leur longueur totale a varié entre $18 \mathrm{~m}$ et $21 \mathrm{~m}$, dont $9 \mathrm{~m}$ de scellement sous injection IGU (Recommandations TA 95). Leur charge de service est de $450 \mathrm{kN}$, étant bien entendu qu'ils sont testés à $20 \%$ au-dessus de cette valeur et ensuite bloqués.

- Viennent ensuite plusieurs nappes de clous métalliques constítués d'armatures $\Phi 32 \mathrm{~mm}$ et de longueur $12 \mathrm{~m}$ : on a choisi pour la commodité et la sécurité des excavations de ne pas dépasser un espacement vertical de $2 \mathrm{~m}$, l'espacement horizontal des clous étant compris entre $1 \mathrm{~m}$ et $2 \mathrm{~m}$ selon le cas de charge.

La stabilité globale de la fouille confortée fut étudiée à l'état limite ultime par la méthode des tranches (Bishop) en adoptant pour les caractéristiques des matériaux utilisés des coefficients de sécurité définis dans CLOUTERRE 91, le coefficient de sécurité globale $\Gamma$ min devant être supérieur ou égal à 1.

Le logiciel TALREN, que nous avons utilisé pour un premier chantier de clouage au Liban (et au ProcheOrient) en 1983 (tour Ghazal à Beyrouth), fut à la base de tous les calculs et résultats obtenus.

On donne sur les figures 4, 5, 6, 7,8,9 et 10 les résultats de stabilité globale obtenus pour les principales coupes existantes.

\section{6}

\section{Exécution des travaux}

Les premiers problèmes commencèrent à se poser dès le début du chantier car une partie du terrain naturel se trouvait au-dessus de la cote 0 notamment sur le côté est, dans la zone appelée FG.

Comme le montre la vue en plan le bâtiment luimême qui possède un seul sous-sol est éloigné d'environ $4 \mathrm{~m}$ du parement à découvrir (Figs. 1 et 11). Par ailleurs son rez-de-chaussée est protégé, à la limite de la parcelle, par un mur en béton armé en L posé probablement sur le sol rapporté avec lequel on avait remblayé derrière le sous-sol après son exécution. La tête de ce mur était à $+6,5$ et son pied à $+3,5$.

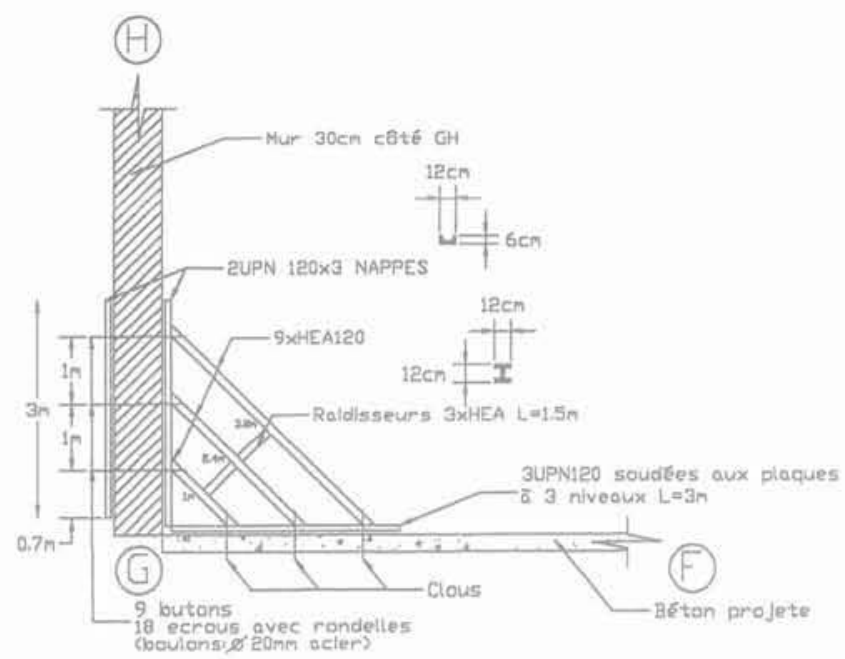

FIG. 15 Renforcement du coin G. Vue en plan Échelle 1/100.

Reinforcement of corner G. Plane view - Scale $1 / 100$

Un clouage avec des clous de $4 \mathrm{~m}$ de longueur fut exécuté avec une densité très forte, de l'ordre de 1 clou par $\mathrm{m}^{2}$, pour la raison simple que le déblai ne tenait à la verticale que sur $1 \mathrm{~m}$ de hauteur au plus. Arrivé à +1 , on s'aperçut que le mur en L avait basculé d'au moins $2 \mathrm{~cm}$. On décida alors de retenir le pied du mur au moyen de poutrelles constituées par des IPN 160 à raison d'une poutre tous les $2 \mathrm{~m}$, ces poutres étant tenues à leur pied par deux poutres en $\mathrm{U}$ retenues par une nappe de tirants précontraints de $15 \mathrm{~m}$ de longueur chacun. Les figures 12 et 13 donnent une verification du clouage réalisé et une coupe du confortement exécuté.

Sur le côté perpendiculaire appelé GH il existait un mur de parking dont la tête est à $+7,20$ et le pied à $-3,15$. Là aussi pour éviter tout déplacement important de ce mur on a prévu des poutrelles IPN 200 espacées de $2 \mathrm{~m}$, dont la tête est à +1 et bloquées à $-3,5$ par une nappe de tirants précontraints de longueur $12 \mathrm{~m}$ espacés de $3 \mathrm{~m}$ et constitués de monobarre DYWIDAG 32 nuance 900/1030, dont la charge de service est de

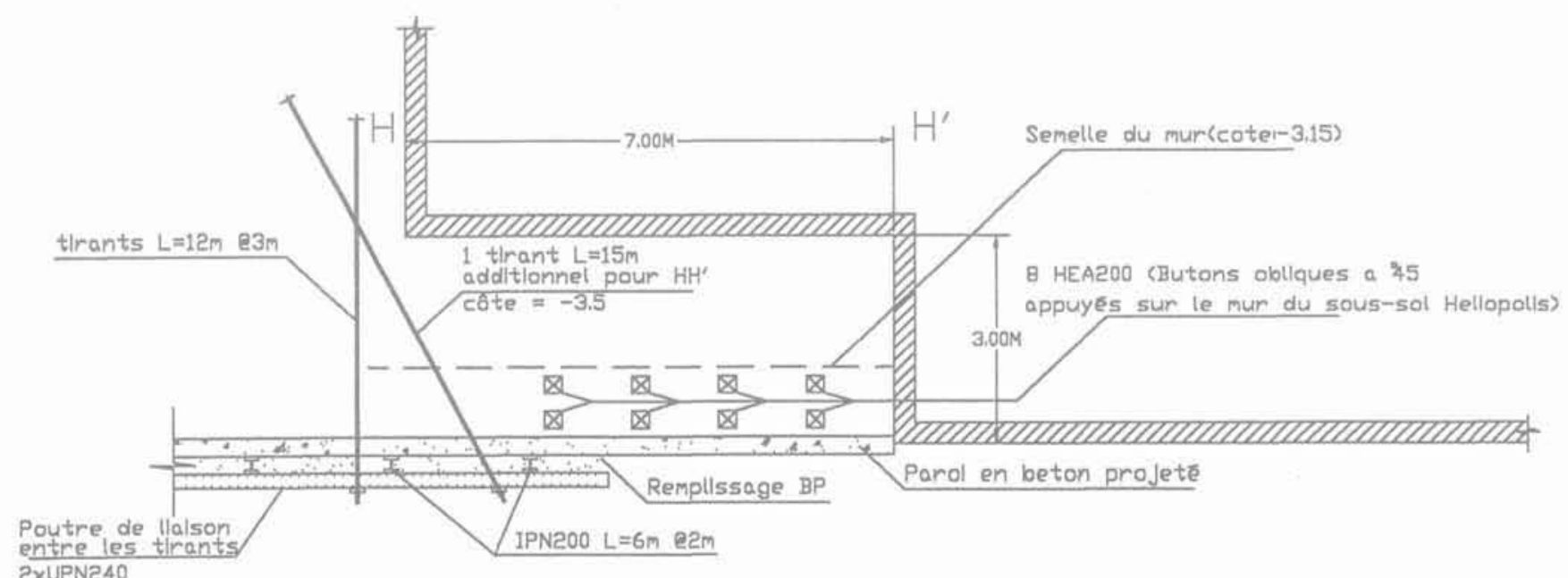
2XUPN240

FIG. 16 Coupe - Échelle 1/100. Section - Scale 1/100 


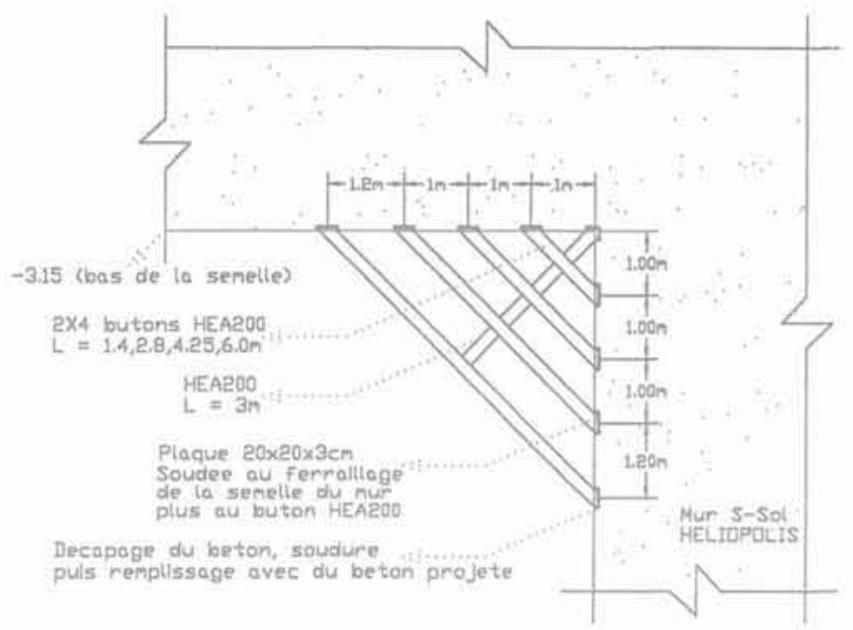

FG. 17 Élévation $\mathrm{H}-\mathrm{H}^{\prime}$. Échelle 1/100. Elevation $\mathrm{H}-\mathrm{H}^{\prime}$. Scale $1 / 100$.

$450 \mathrm{kN}$ (Fig. 14). Au coin des parties FG et GH, on mit en place des butons constitués par des HEA 120 et reliés entre eux par des raidisseurs (Fig. 15).

Le même type de confortement fut utilisé dans la partie $\mathrm{HH}^{\prime}$ où le mur de soutènement des sous-sols du bâtiment Héliopolis présentait un décrochement ren-

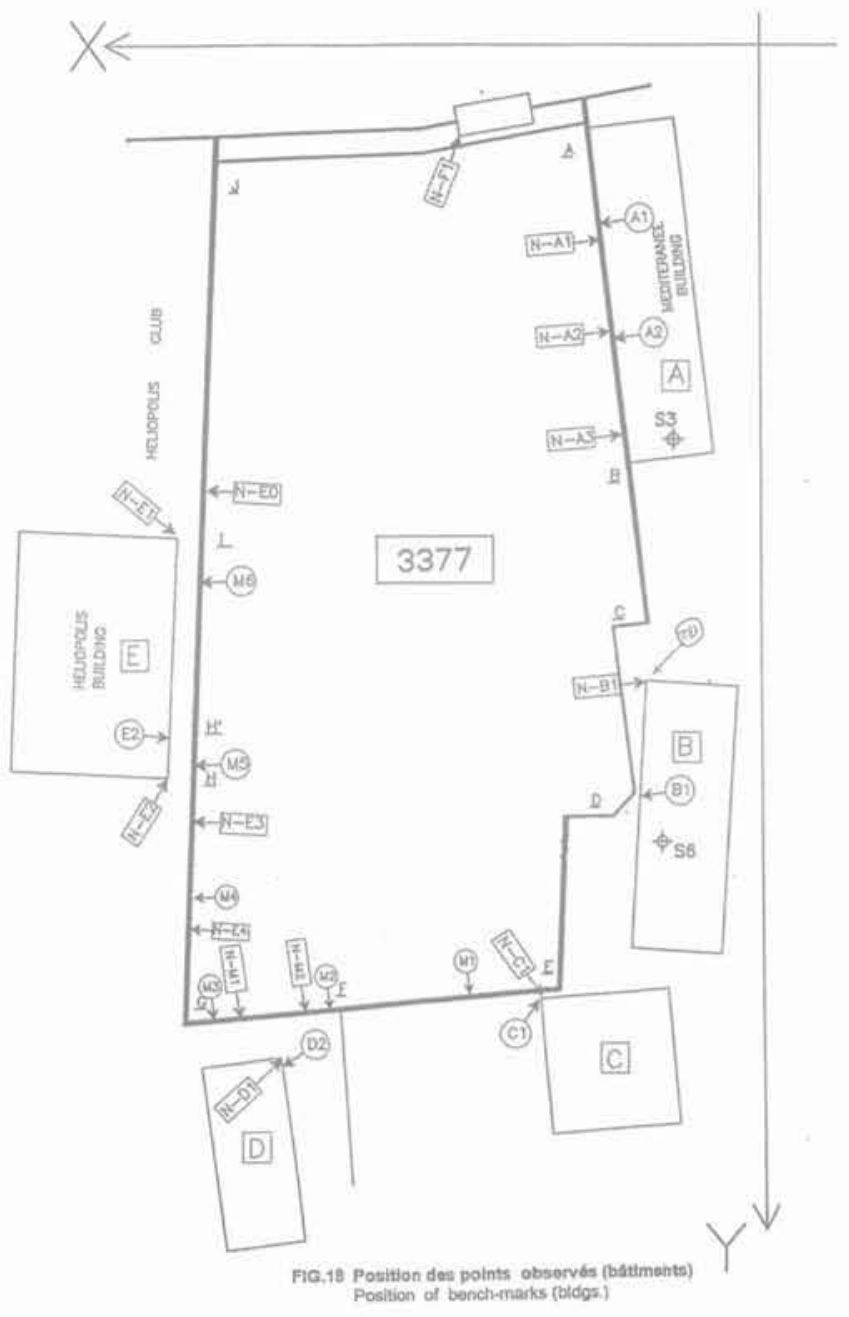

FG. 18 Position des points observés (bâtiments). Position of bench-marks (bidgs). dant nécessaire son soutènement par un clouage avec des clous de faible longueur, avec une densité de deux clous $/ \mathrm{m}^{2}$, associé à une nappe de tirants précontraints et à des poutrelles métalliques (Figs. 16 et 17).

- A ce stade furent installés des repères au pied et en tête des bâtiments existants A, B, C, D et E.

Ensuite, au fur et à mesure que l'on atteignait et dépassait le niveau de fondations et que des tirants étaient mis en tension, on complétait à leur niveau l'installation des repères à viser. Les figures 18 et 19 montrent la position des points observés durant l'ouverture de la fouille.

- Les premiers déplacements furent observés au niveau de la première nappe de tirants sous l'immeuble B. On mesura alors sur les trois repères installés un déplacement horizontal compris entre $5 \mathrm{~mm}$ et $10 \mathrm{~mm}$ au moment où l'on déblayait pour descendre à -13.

Une semaine plus tard, deux autres tirants se déplacèrent horizontalement de 3 à $4 \mathrm{~mm}$, et l'on nota un tassement de 2,7 mm en pied de l'immeuble et un basculement côté fouille au sommet de $4 \mathrm{~mm}$.

Une semaine plus tard, les travaux étant arrêtés à ce niveau, on nota un déplacement supplémentaire des tirants T5, T6 et T6" de $2 \mathrm{~mm}$ à $8 \mathrm{~mm}$ et un tassement en un autre point du bâtiment $\mathrm{B}$ égal à $3 \mathrm{~mm}$.

Le 27 mai 1998, on était donc à la cote -13 avec les mouvements suivants sous le bâtiment B :

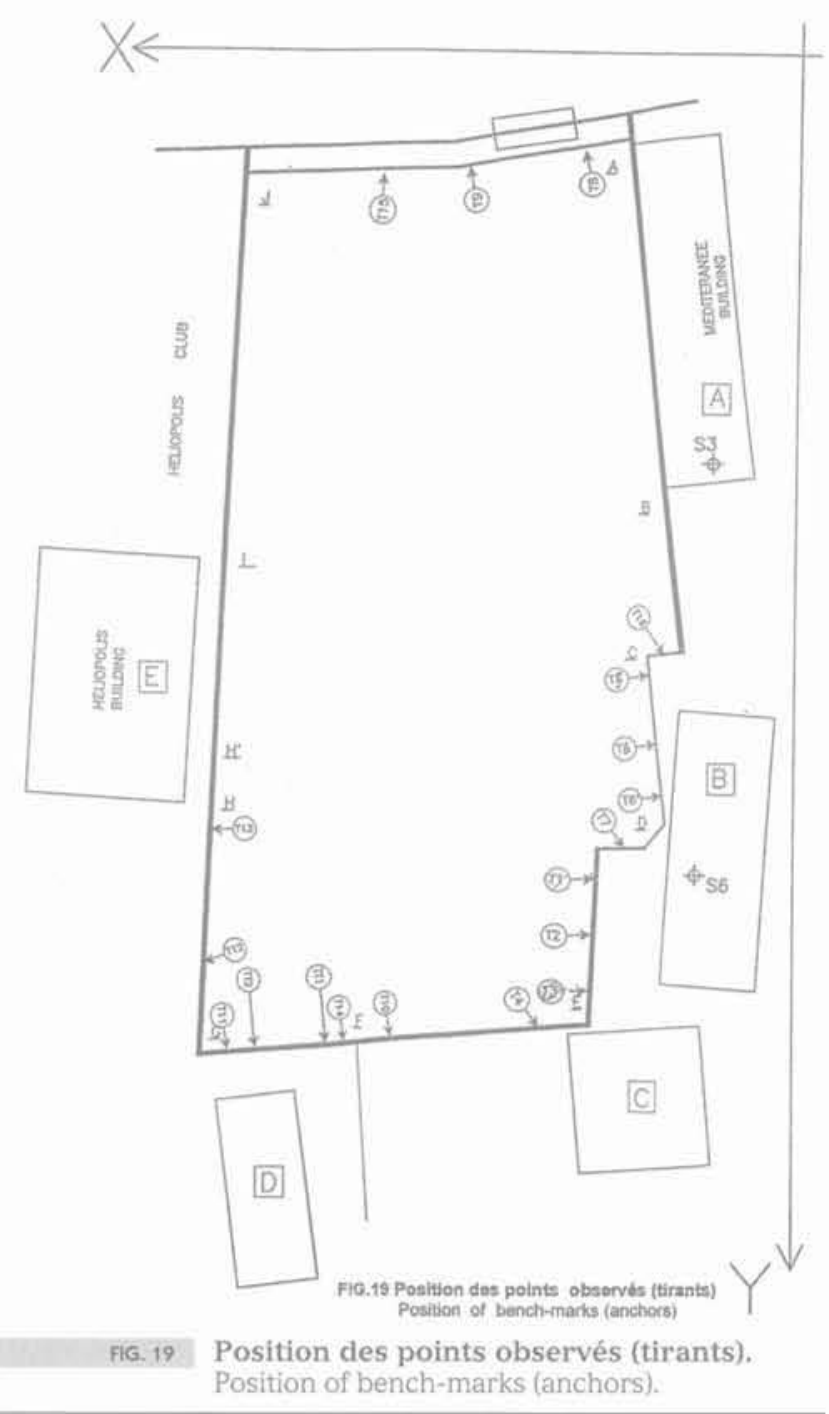


Déplacement horizontal de la première nappe de tirants :

$\begin{array}{ll}\text { T5 } & 7 \mathrm{~mm} \\ \text { T6 } & 10 \mathrm{~mm} \\ \text { T6 }^{\prime} & 10 \mathrm{~mm}\end{array}$
$3 \mathrm{~mm}$

Tassement vertical mesuré au pied du bâtiment

Basculement vers la fouille mesuré au sommet du bâtiment $4 \mathrm{~mm}$.

On vérifia alors que le tassement différentiel en résultant était de 0,75 pour mille, ce qui était encore loin du maximum admissible pour ce type de bâtiment, et que la rotation était de l'ordre de 0,11 pour mille. Ces déplacements ne pouvaient pas provoquer des fissures dans le bâtiment. A ce stade, la fouille avait une hauteur de $15,8 \mathrm{~m}$.

D'après CLOUTERRE le déplacement horizontal et vertical prévisible en l'absence de tirants précontraints est compris entre $16 \mathrm{~mm}$ (semi-rocheux) et $32 \mathrm{~mm}$ (sable). Les formations traversées étant en général du sable moyennement cimenté, on se situait probablement entre ces deux valeurs.

On constata alors que le déplacement observé était inférieur à la moitié des valeurs prévisibles, cela étant dû à la présence des tirants précontraints.

- Le maître d'œuvre décida, par précaution supplémentaire, de réaliser une nappe de tirants précontraints de $15 \mathrm{~m}$ de longueur à la cote -13 pour éviter d'avoir un supplément de déplacement et limiter la décom- pression du sol en dessous de -13 . Cette décision fut étendue au parement devant les bâtiments $C$ et $D$.

- En cours d'exécution des travaux, les études de la tour progressaient et l'on fut averti que ses fondations, sur radier général de 2,25 m d'épaisseur, seraient à $-20,25$. Il était donc nécessaire, dans la zone qui avait accusé des mouvements certains, c'est-à-dire essentiellement au voisinage du bâtiment B, de descendre la fouille de $2,25 \mathrm{~m}$. La stabilité de la fouille était précaire pour cette hauteur supplémentaire car la distance qui séparait l'extrémité du radier du parement de la fouille était inférieure à $2 \mathrm{~m}$.

On prit donc la décision de rajouter une nappe de tirants précontraints de $15 \mathrm{~m}$ de longueur chacun, espacés de $1 \mathrm{~m}$ au pied du parement initial et une autre à mi-hauteur de la fouille supplémentaire pour le radier, cette dernière étant constituée par des tirants précontraints de $12 \mathrm{~m}$ de longueur espacés de $1,5 \mathrm{~m}$ ou de $2,0 \mathrm{~m}$ suivant la distance du parement cloué au radier. Les figures 20,21 et 22 ont donné les résultats du calcul de stabilité globale dans ce cas.

- Au milieu du mois d'août 1998 on atteignit la cote -18 et dans certaines zones la cote basse du radier à $-20,25$. On nota alors que le point B1 situé au haut de l'immeuble B avait bougé de $6 \mathrm{~mm}$ de plus vers la fouille, totalisant ainsi un déplacement horizontal global de $10 \mathrm{~mm}$. La rotation globale du bâtiment était maintenant de 10/30000 c'est-à-dire voisine de 0,35 pour mille, de l'ordre du dixième de la valeur admissible, mais cela ne pouvait créer des fissures dans le bâtiment et fut vérifiée par les observations sur place.

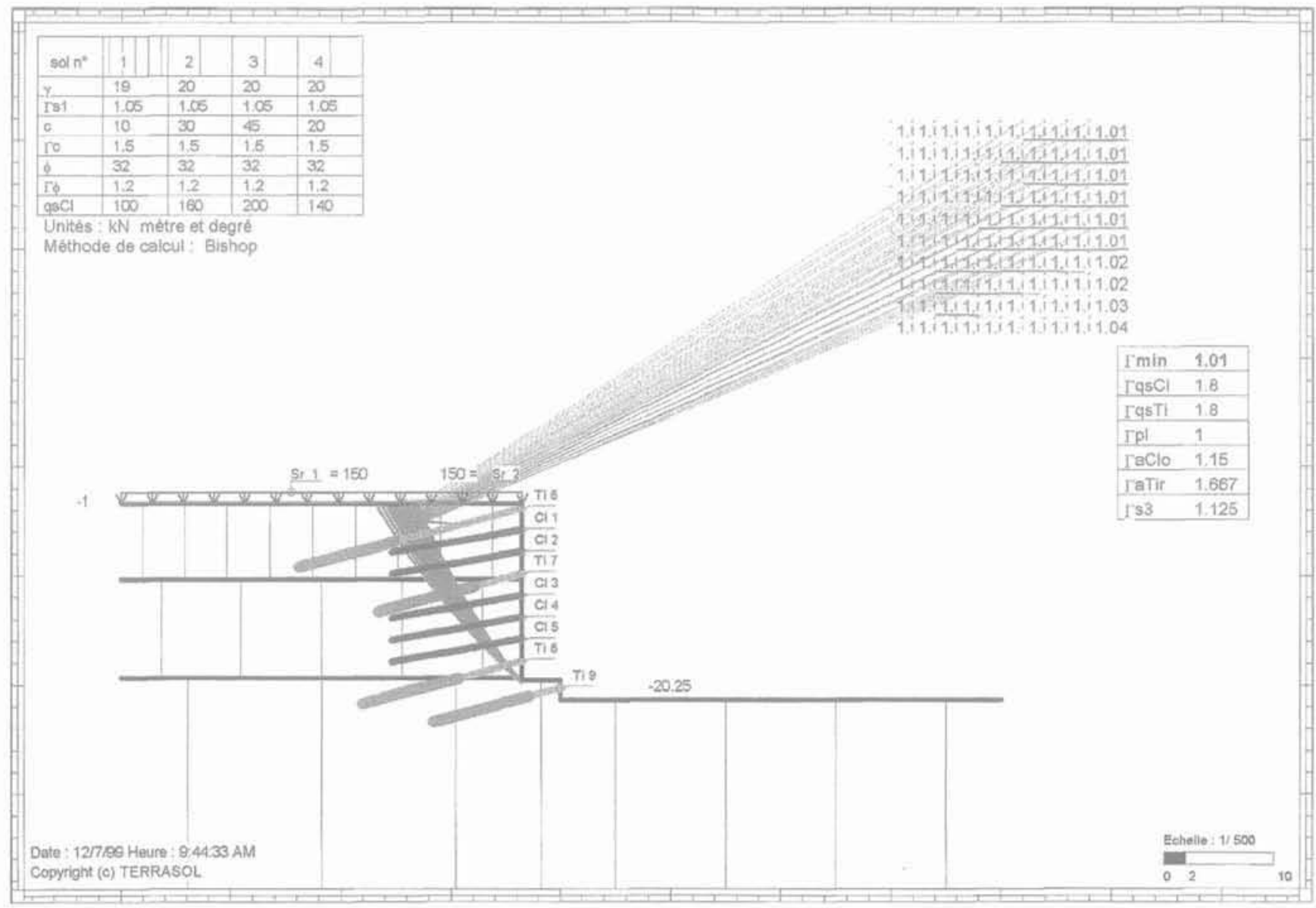



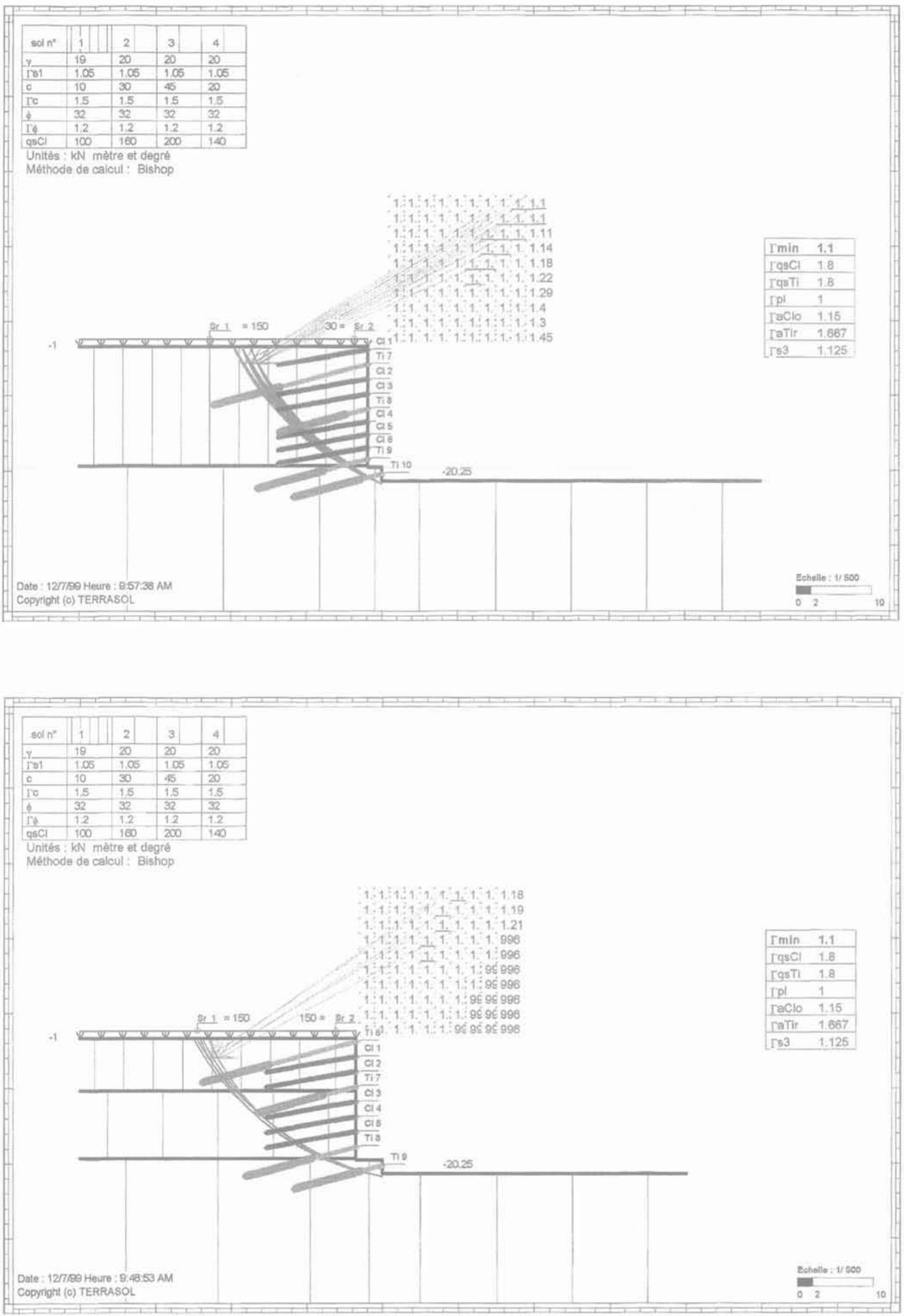
A quoi était dû ce déplacement supplémentaire malgré toutes les précautions prises pour le bloquer? Probablement à un éboulement important survenu au coin $\mathrm{E}$ au passage entre -16 et -18 , cet accident ayant provoqué l'écoulement de quelques 2 à $3 \mathrm{~m}^{3}$ de sable. Le dernier mouvement observé fut de ce côté de la fouille et l'on prit la décision, en cours de réalisation du mur périphérique du bâtiment et au cas où les poutres de solidarisation constitueraient un obstacle devant ses armatures, d'adopter le processus suivant qui fut respecté scrupuleusement par tranches de trois tirants successifs au plus :

- débloquer les tirants ;

- sectionner la poutre métallique en U correspondante et l'enlever;

- remettre les tirants à une tension voisine de leur tension d'origine en s'appuyant sur le béton projeté directement.

- Côté EF, où les contraintes verticales exercées étaient faibles puisque correspondant à deux sols seulement. on nota à -18 m un déplacement de $14 \mathrm{~mm}$ pour le tirant T4 et de $16 \mathrm{~mm}$ pour le tirant T10.

Que s'était-il produit pour ce parement? A la cote $-16,5$ c'est-à-dire à $1,5 \mathrm{~m}$ au-dessus du fond de la fouille, les avoisinants exigèrent l'arrêt du clouage après qu'ils eurent constaté des fissures dans leur soussol.

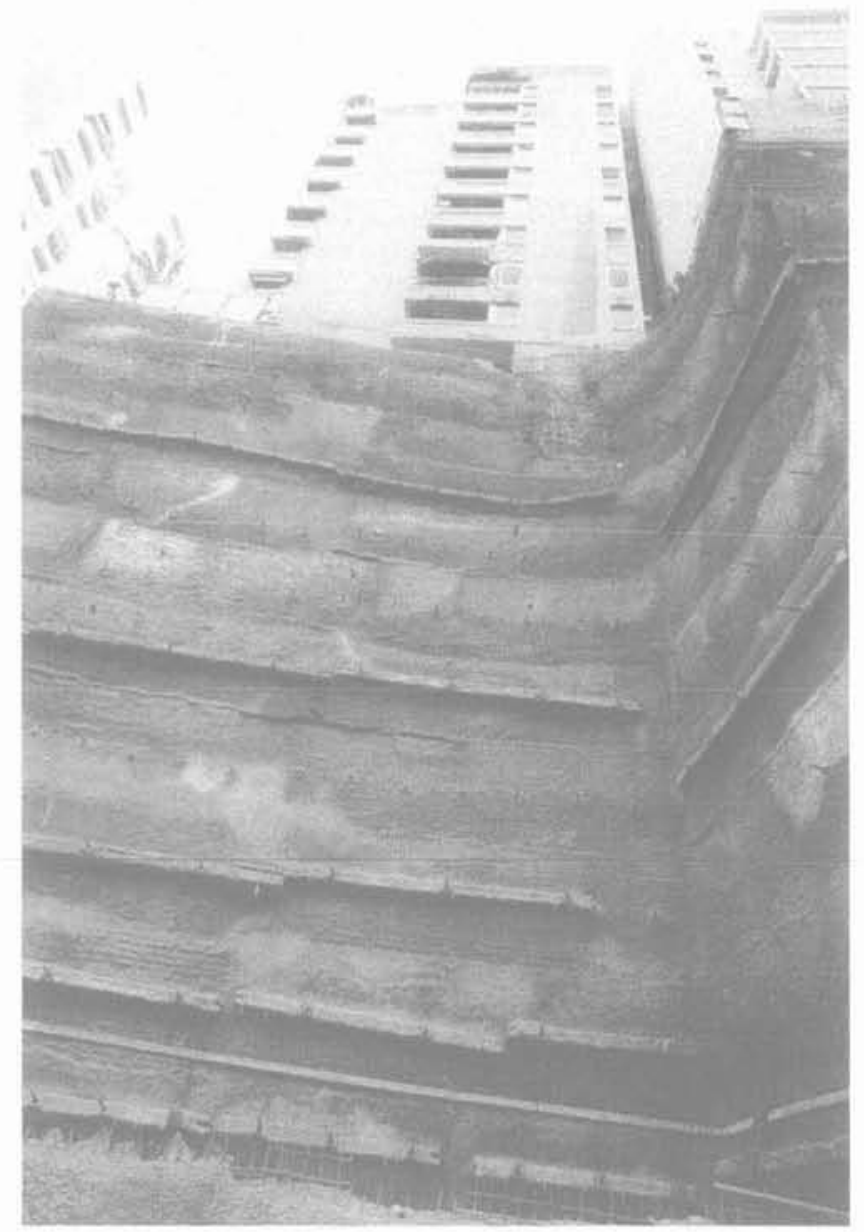

FIG. 24 Zone CD (Imm. JOUMBLATT : sans recul) 9 étages - 1 R.D.C -1 sous-sol.

Zone CD (JOUMBLATI bldg. : no retreat) 9 stories -1 ground floor -1 basement.

L'examen de ces fissures montra qu'elles s'étaient produites uniquement dans les cloisons et leur position laissant plutôt croire à un tassement car ces cloisons étaient posées directement sur le carrelage sans aucune fondation. Elles auraient mème pu tasser de ce fait avant les travaux de soutènement. Cette interprétation était fortement corroborée par le fait qu'aucun déplacement dans les tirants n'avait été mesuré jusqu'à la cote $-16,5$. Toutefois, pour autoriser la poursuite des travaux, il fut exigé que la fouille soit soutenue à partir de $-16,5$ par des pieux de $40 \mathrm{~cm}$ de diamètre retenus par une nappe de tirants précontraints (Fig. 19).

Le système retenu présentait ainsi une plus grande rigidité que le parement en béton projeté. Pourquoi donc y avait-il eu ce déplacement? L'explication la plus probable tient à la méthode d'exécution des pieux: en effet, en cours de forage à la tarière, il se produit un éboulement en partie haute du trou qui exige la mise en place d'un tubage de protection permanent à l'intérieur duquel le béton est coulé.

Ce tubage ne colle pas exactement à la surface du sol côté parement. Il se produit une décompression et il en résulte un déplacement obligatoire du sol de l'ordre du centimètre pour lui permettre de s'appuyer sur le pieu et de mobiliser la butée aval. Ce déplacement génère des mouvements en partie haute et explique le
FG. 23 Zone AB (Banque de la Méditerranée) 13 étages -1 R.D.C. -2 sous-sols.

Zone $\mathrm{AB}$ (Banque de la Méditerranée) 13 stories -1 ground floor -2 basements. 


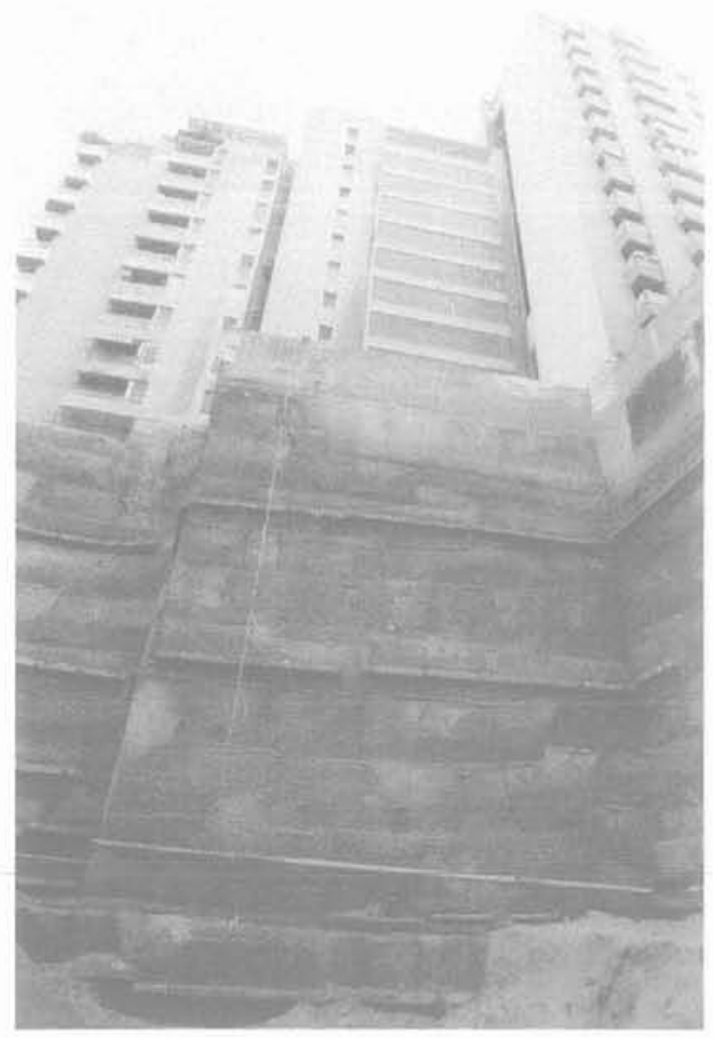

FIG, 25 Zone DE (Imm, JOUMBLATT : avec

9 étages -1 R.D.C. -1 sous-sol. Zone DE (JOUMBLATT bidg, : with retreat) 9 stories - 1 ground floor - 1 basement.

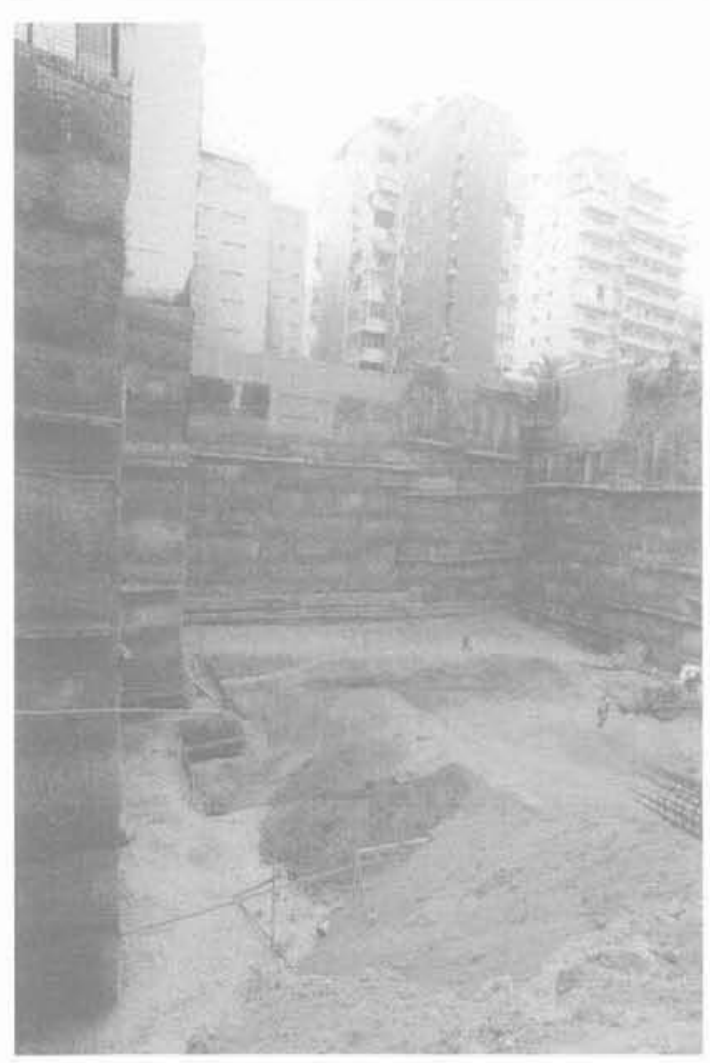

F16. 27 Zone FG (Imm. JABER) 10 étages + 1 R.D.C + 1 sous-sol,

Zone FG (JABER bldg.) 10 stories + 1 ground floor +1 basement.

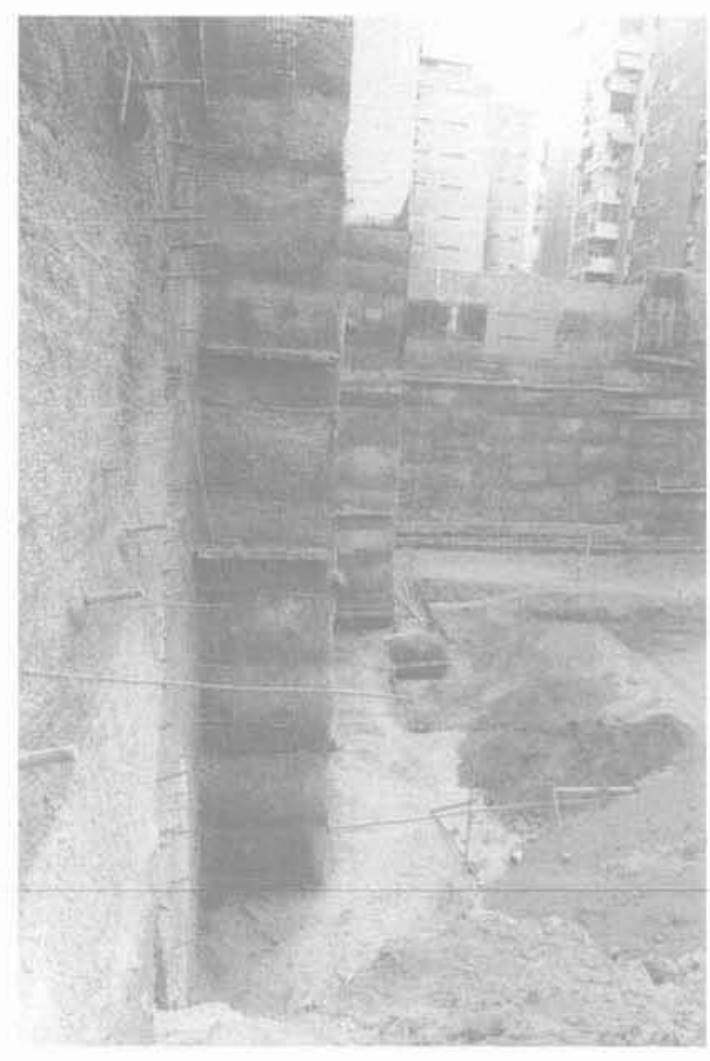

FG. 26 Zone EF (dépôt + parking) 2 soussols.

Zone EF (warehouse + parking) 2 basements.

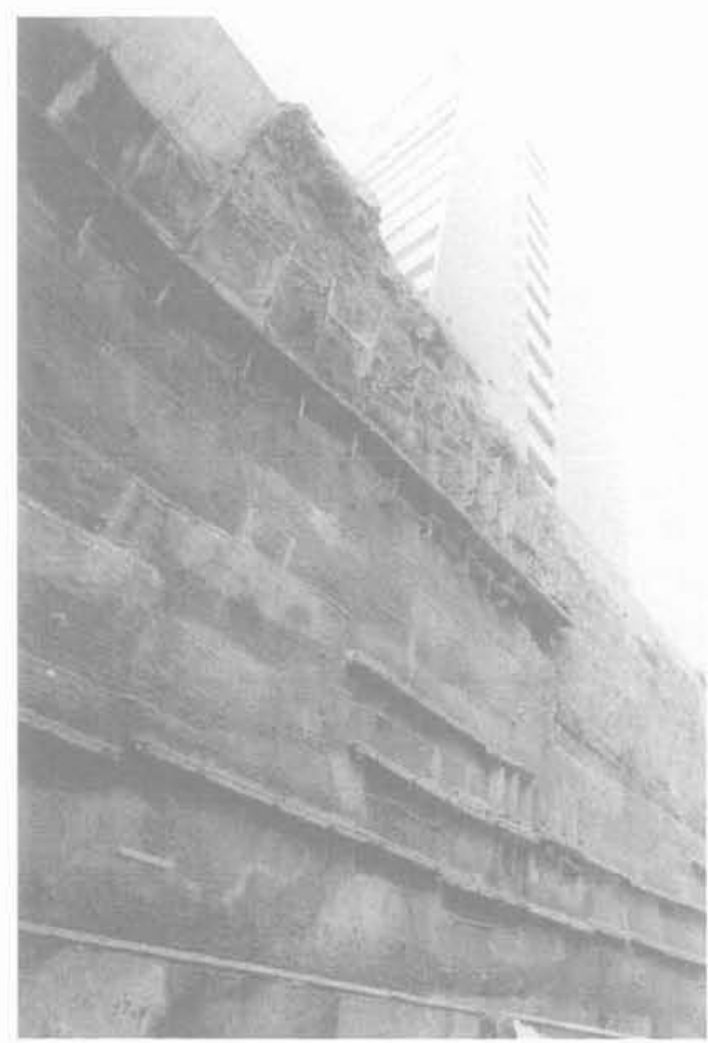

FIQ.28 Zone GHI (Imm. HELIOPOLIS) 16 étages +1 R.D.C. +2 sous-sols. Zone GHI (HELIOPOLIS bldg.) 16 stories + 1 ground floor + 2 basements. 
déplacement des tirants. Là aussi ce furent les derniers mouvements observés.

- Enfin côté clôture et bâtiment E (HELIOPOLIS), on n'enregistra de mouvement que lorsqu'on atteignit la cote $-18,2$ :

- sous la clôture les déplacements horizontaux et verticaux des tirants étaient respectivement égaux à $13 \mathrm{~mm}$ et $5 \mathrm{~mm}$;

- sous l'immeuble lui-même ces mêmes déplacements de tirants furent limités à $12 \mathrm{~mm}$ et $3 \mathrm{~mm}$ alors qu'au niveau du bâtiment, on notait un tassement négligeable de $2 \mathrm{~mm}$ et un déplacement horizontal au sommet de $9 \mathrm{~mm}$ correspondant à une rotation de 0,2 pour mille, acceptable et non préjudiciable à la structure.

- Le cas du bâtiment A constitua un cas spécial de soutènement. En effet, les propriétaires de cet immeuble quí abrite la Banque Méditerranée arguant du fait qu'il était sous-dimensionné et qu'il présentait des fissures bien avant que l'on commence à excaver refusèrent la mise en place de tirants (actifs ou passifs) sous leur bâtiment à moins que le soutènement ne soit constitué par un écran de pieux jonctifs de $40 \mathrm{~cm}$ de diamètre, encastré de $3 \mathrm{~m}$ dans le sol, et retenu par trois nappes de tirants précontraints dont la densité était très importante : espacement horizontal de $1,2 \mathrm{~m}$ pour toutes les nappes, longueur de $18 \mathrm{~m}$ pour la nappe supérieure, de $15 \mathrm{~m}$ pour la nappe intermédiaire, et de $12 \mathrm{~m}$ pour la nappe inférieure.
Les figures $23,24,25,26,27$ et 28 montrent les différentes zones traitées une fois la cote $-20,25$ atteinte.

\section{7}

\section{Conclusion}

Le procédé de renforcement des sols par inclusions s'est avéré être efficace, même dans un contexte géologique et urbanistique très sensible.

Cela a exigé le respect de quelques consignes simples, mais à appliquer strictement : suivre constamment l'exécution au moyen du logiciel Talren en modifiant si nécessaire les données sur le sol au fur et à mesure de l'approfondissement de l'excavation, ouvrir par panneaux bien limités, ausculter de manière permanente et régulière les bâtiments limitrophes et les tirants précontraints installés.

\section{REMERCIEMENTS}

L'auteur remencie vivement ses collaborateurs, ingénieurs et techniciens, dont certains l'accompagnent depuis plus de vingt ans, sans lesquels ce travail délicat n'aurait pas pu s'accomplir.

11 exprime aussi sa gratitude envers le promoteur, monsieur $A$ il Ahmad, qui lui a accordé une confiance totale en prenant le risque d'accepter une solution non classique pour la réalisation du soutènement de la fouille. 